\title{
On quantum model of supergravity compensator
}

\author{
I.L.Buchbinder and A.Yu.Petrov \\ Department of Theoretical Physics \\ Tomsk State Pedagogical University \\ Tomsk 634041, Russia
}

\begin{abstract}
A new $N=1$ superfield model in $D=4$ flat superspace is suggested. This model describes dynamics of chiral compensator and can be treated as a low-energy limit of $D=4, N=1$ quantum superfield supergravity. Renormalization structure of this model is studied and one-loop counterterms are calculated. It is shown that the theory is infrared free. An effective action for the model under consideration is investigated in infrared domain. The lower contributions to the one-loop effective action are computed in explicit form.
\end{abstract}

1. It is well known that four-dimensional simple supergravity can be formulated in $N=$ 1 curved superspace in terms of vector superfield $H^{m}(z)$ and chiral $\Phi(z)$ and antichiral $\bar{\Phi}(z)$ compensators [1-3] (see also [4]). Here as usual $z^{m} \equiv\left(x^{m}, \theta^{\mu}, \bar{\theta}^{\dot{\mu}}\right)$ are the superspace coordinates. General approach to quantum superfield supergravity has been developed in refs. $[5,6]$. However a number of concrete quantum aspects has not been investigated in details because of complicated enough structure of the superfield supergravity itself.

Our goal in the present paper is to formulate a simplified model associated with $N=1, D=4$ superfield supergravity and describing a dynamics only of compensator superfields in flat superspace. We show that this model makes possible to carry out a detailed investigation of many quantum aspects and possessses remarkable properties in infrared domain. It allows to treat such a model as a natural low- energy limit of quantum superfield supergravity with matter.

The model under consideration is based on an idea of induced gravity. An approach to four-dimensional quantum gravity model induced by conformal anomaly of matter fields in curved space-time has been suggested in ref. [6]. It has been shown that this model is super-renormalizable in infrared limit and infrared free. In the given paper we present a supersymmetric model of induced quantum gravity and investigate its quantum aspects.

2. We start with known superconformal anomaly in $N=1$ curved superspace. This anomaly is formulated in terms of supercurrent and supertrace [7,8] (see also [4]) which are the superfield analogs of the energy-momentum tensor and its trace respectively. For superconformally invariant theories the classical supertrace $V$ vanishes. However in quantum theory the supertrace $V$ is not equal to zero because of superconformal anomaly and can be written as follows $[9,10,4]$

$$
V_{A}=-(4 \pi)^{-2}\left(a W^{2}+b G+c\left(\bar{D}^{2}-8 R\right) D^{2} R\right)
$$


Here $a, b, c$ are some numbers depending on the field content of the theory, $W^{2}=$ $W^{\alpha \beta \gamma} W_{\alpha \beta \gamma}, G=W^{2}-\frac{1}{4}\left(\bar{D}^{2}-8 R\right)\left(G^{a} G_{a}+2 R \bar{R}\right), D_{A}=\left(D_{a}, D_{\alpha}, \bar{D}_{\dot{\alpha}}\right)$ are the supercovariant derivatives; $R, G_{a}, W_{\alpha \beta \gamma}$ are superfield strengths expressed in terms of supergravity prepotentials $H^{m}, \Phi, \bar{\Phi}$; (we use the denotions accepted in ref. [4]).

A superfield action leading to the anomaly (1) has been found in ref. [11]. Our goal is to investigate a model a total action of which is a sum of the anomaly generating action [11] and the action of $N=1, D=4$ superfield supergravity (see [4]). Being transformed to conformally flat superspace the total action of our model takes the form

$$
\begin{aligned}
S & =\int d^{8} z\left(-\frac{Q^{2}}{2(4 \pi)^{2}} \bar{\sigma} \square \sigma+\bar{D}^{\dot{\alpha}} \bar{\sigma} D^{\alpha} \sigma \times\right. \\
& \left.\times\left(\xi_{1} \partial_{\alpha \dot{\alpha}}(\sigma+\bar{\sigma})+\xi_{2} \bar{D}_{\dot{\alpha}} \bar{\sigma} D_{\alpha} \sigma\right)-\frac{m^{2}}{2} e^{\sigma+\bar{\sigma}}\right)+\left(\Lambda \int d^{6} z e^{3 \sigma}+\text { h.c. }\right)
\end{aligned}
$$

Here we use the flat supercovariant derivatives $D_{\alpha}, \bar{D}_{\dot{\alpha}}, \partial_{\alpha \dot{\alpha}} ; \sigma=\ln \Phi, \Lambda$ is a cosmological constant $m^{2}=\frac{6}{\kappa^{2}}$ where $\kappa$ is the gravitational constant; the $Q^{2}, \xi_{1}, \xi_{2}$ are expressed in terms of $a, b, c$. We will consider the $Q^{2}, \xi_{1}, \xi_{2}, m^{2}, \Lambda$ as the arbitrary and independent parameters of the model. The superfield model with the action (2) is the basic object of this paper. We are going to investigate a renormalization structure of the model (2), compute the one-loop counterterms, study a behaviour of running couplings and calculate one-loop effective action.

3. To clarify a renormalization structure and calculate the counterterms we should find the superpropagator and vertices for the model (2). This model is characterized by the matrix superpropagator with the matrix elements $G_{++}, G_{+-}, G_{-+}, G_{--}$where the sign + corresponds to chiral field and the sign - to the antichiral one. The explicit form of above matrix is given by the quadratic part of the action (2) and can be found easy enough. The model under consideration has the vertices of the four types: $V_{1}=$ $\xi_{1} \bar{D}^{\dot{\alpha}} \bar{\sigma} D^{\alpha} \sigma \partial_{\alpha \dot{\alpha}}(\sigma+\bar{\sigma}), V_{2}=\xi_{2} \bar{D}_{\dot{\alpha}} \bar{\sigma} \bar{D}^{\dot{\alpha}} \bar{\sigma} D^{\alpha} \sigma D_{\alpha} \sigma, V_{3}=-\frac{m^{2}}{2}\left(e^{\sigma+\bar{\sigma}}-1-(\sigma+\bar{\sigma})-\frac{1}{2}(\sigma+\bar{\sigma})^{2}\right)$, $V_{4}=\Lambda\left(-\frac{D^{2}}{4 \square}\right)\left(e^{3 \sigma}-1-3 \sigma-\frac{9}{2} \sigma^{2}\right)+\bar{\Lambda}\left(-\frac{\bar{D}^{2}}{4 \square}\right)\left(e^{3 \bar{\sigma}}-1-3 \bar{\sigma}-\frac{9}{2} \bar{\sigma}^{2}\right)$. All vertices correspond to the action written as an integral over whole superspace.

A renormalization structure of the theory is defined in terms of superficial degree of divergences $\omega$. For explicit calculation of the $\omega$ and the counterterms we will use the supergraph technique (see [12,4]) specially generalized for use namely in our model (2). A value of the $\omega$ is caused by the $D$-factors and momentum dependence of the superpropagator, by the form of vertices and by integration over momenta in the supergraphs. The total contribution to $\omega$ from superpropagators, vertices and integrations looks like this $\omega \leq 2 V_{1,2}-2 L_{2}-5 L_{1}+2 L-V_{4}$ where $V_{1,2,4}$ are numbers of vertices of the type $V_{1,2}$ and $V_{4}$ respectively in supergraph, $L_{1}$ is a number $G_{++}, G_{--}$-lines, $L_{2}$ is a number of $G_{+--}, G_{-+}$-lines and $L$ is a number of loops in the supergraph. Taking into account the identities $L+V-P=1, V=V_{1}+V_{2}+V_{3}+V_{4}$ where $P$ is a number of all internal lines we obtain the final result for $\omega$.

$$
\omega \leq 2-3 L_{1}-2 V_{3}-3 V_{4}
$$

The condition of divergence $\omega \geq 0$ leads to $L_{1}=0, V_{4}=0, V_{3}=0,1$. Hence the divergent diagrams cannot contain the $V_{4^{-}}$type vertices and $G_{++^{-}}, G_{--}$-type lines. They can include no more that one vertex of $V_{3}$-type. We see a number of divergent structures is more 
restricted in compare with non-supersymmetric case [6]. In particular we have a nonrenormalization theorem: the vertex of $V_{4}$-type is always finite.

Thus, the divergent supergraphs can include an arbitrary number of $G_{+_{-}-}, G_{-_{+}}$-lines and arbitrary number of $V_{1,2}$-type vertices. But if $\xi_{1}=\xi_{2}=0$ then the vertices of $V_{1,2^{-}}$ type are absent at all. The only vertices which can present in divergent diagrams are now the $V_{3}$-type ones. But their number is exactly equal to 1 and corresponds only to one-loop supergraphs. It means, at $\xi_{1}=\xi_{2}=0$ the theory becomes to be super-renormalizable. We will show that in infrared limit the running couplings $\xi_{1}(t)$ and $\xi_{2}(t)$ tend to zero and namely above case is realized.

4. Let us investigate the one-loop renormalizability of the model (2). We start with calculations of the renormalization constants for the parameters $\xi_{1}, \xi_{2}$ and $Q^{2}$. The corresponding supergraphs are given by the Fig.1, Fig.2 and Fig.3

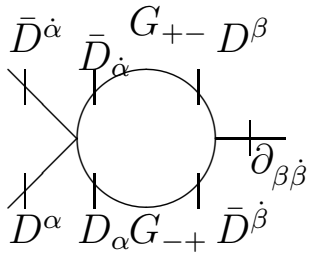

Fig.1

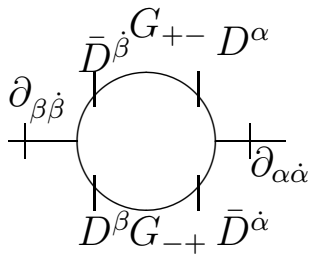

Fig.3

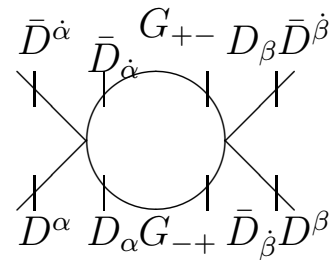

Fig.2

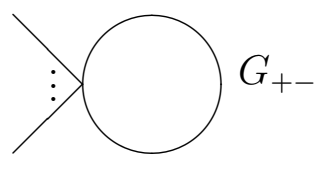

Fig.4

The straightforward calculations of the divergent parts of these supergraphs within dimensional reduction scheme (see f.e. [4]) allow to find the renormalization transformations in the form

$$
\begin{aligned}
Q_{(0)}^{2} & =\mu^{-\epsilon} Z_{Q} Q^{2}, \quad \xi_{(0) 1,2}=\mu^{-\epsilon} Z \xi_{1,2} \\
Z_{Q} & =1+\frac{2^{14} 3^{2} \pi^{6} \xi_{1}^{2}}{Q^{6} \epsilon}, \quad Z=1+\frac{2^{11} 3^{2} \pi^{2} \xi_{2}}{Q^{4} \epsilon}
\end{aligned}
$$

where $\epsilon=4-d$ is a parameter of dimensional regularization and $\mu$ is an arbitrary parameter of mass dimension, $Q_{(0)}^{2}, \xi_{1(0)}, \xi_{2(0)}$ are the bare parameters and $Q^{2}, \xi_{1}, \xi_{2}$ are the renormalized ones. We see that in one-loop approximation there is the same renormalization constant $Z$ both for $\xi_{1}$ and $\xi_{2}$. It means in particular, if we put $c \xi_{1(0)}=$ $\xi_{2(0)}$ with some constant $c$ then the renormalized parameters $\xi_{1}$ and $\xi_{2}$ satisfy the same relation $c \xi_{1}=\xi_{2}$. One-loop renormalization does not destroy relationship between these parameters.

Let us consider a renormalization of the parameter $m^{2}$ at $\xi_{1}=\xi_{2}=0$. As we will see, namely this situation is realized in infrared limit. The divergent supergraphs contributing to renormalization of $m^{2}$ include the only vertex of $V_{3}$-type, the only $G_{+-}$-type internal line and an arbitrary number of the external lines of the $\sigma$ and $\bar{\sigma}$ superfields. The 
corresponding supergraphs are given by Fig.4. The straightforward calculations lead to

$$
m_{0}^{2}=Z_{m^{2}} m^{2} ; \quad Z_{m^{2}}=1+\frac{2}{Q^{2} \epsilon}
$$

Here $m_{0}^{2}$ is a bare parameter and $m^{2}$ is a renormalized one.

The eqs. $(4,5)$ define the one-loop renormalization of the model. As for the parameter $\Lambda$ in (2), it was already noted that all supergraphs containing the vertices of $V_{4}$-type are finite. It means that the coupling $\Lambda$ is not renormalized. The fields $\sigma$ and $\bar{\sigma}$ are not renormalized as well.

5. We consider the behaviour of the running couplings in the model (2). The corresponding renormalization group equations can be found on the base of $(4,5)$.

The equations for running constants $\xi_{1}(t), \xi_{2}(t), Q^{2}(t)$ look like this

$$
\frac{d \xi_{1}}{d t}=a \frac{\xi_{1} \xi_{2}}{Q^{4}} ; \frac{d \xi_{2}}{d t}=a \frac{\xi_{2}^{2}}{Q^{4}} ; \frac{d Q^{2}}{d t}=b \frac{\xi_{1}^{2}}{Q^{4}}
$$

where $a=2^{11} 3^{2} \pi^{2}, b=3^{2} 2^{14} \pi^{4}$. The solutions of these equations have the form

$$
\begin{aligned}
\xi_{1}(t) & =\frac{\xi_{1}}{\xi_{2}} \xi_{2}(t) \\
Q^{2}(t) & =Q^{2}+8 \pi^{2} \frac{\xi_{1}^{2}}{\xi_{2}^{2}}\left(\xi_{2}(t)-\xi_{2}\right) \\
t & =\frac{1}{2^{11} 3^{2} \pi^{2}}\left\{-\left[Q^{2}-8 \pi^{2} \frac{\xi_{1}^{2}}{\xi_{2}^{2}}\right]\left(\frac{1}{\xi_{2}(t)}-\frac{1}{\xi_{2}}\right)-\right. \\
& \left.-16 \pi^{2}\left(\frac{\xi_{1}}{\xi_{2}}\right)^{2}\left[Q^{2}-8 \pi^{2} \frac{\xi_{1}^{2}}{\xi_{2}^{2}}\right] \ln \frac{\xi_{2}(t)}{\xi_{2}}+64 \pi^{4}\left(\frac{\xi_{1}}{\xi_{2}}\right)^{4}\left(\xi_{2}(t)-\xi_{2}\right)\right\}
\end{aligned}
$$

Here $\xi_{1,2}=\left.\xi_{1,2}(t)\right|_{t=0}, Q^{2}=\left.Q^{2}(t)\right|_{t=0}$. It is easy to see that in infrared limit $t \rightarrow-\infty$ the $\xi_{1}^{(0)}=\xi_{2}^{(0)}=0$ is an infrared fixed point and $Q^{2}(t) \rightarrow Q^{2}$. This result shows that only supergraphs given by Fig.4 can contribute to $\mathrm{m}^{2}$-renormalization in infrared limit.

Now we consider the running coupling for the parameter $m^{2}$ in infrared domain $\xi_{1}=$ $\xi_{2}=0$. Following to ref.[6] we make the scale transformation $\sigma \rightarrow \alpha \sigma, \bar{\sigma} \rightarrow \alpha \bar{\sigma}$ and $S \rightarrow \frac{1}{\alpha^{2}} S$ where $\alpha$ is a real parameter. The only modification in compare with above consideration is that we should transform the propagator $G_{+-} \rightarrow \alpha^{2} G_{+-}$. It leads to

$$
m_{(0)}^{2}=Z_{m^{2}} m^{2}, \quad Z_{m^{2}}=1+\frac{2 \alpha^{2}}{Q^{2} \epsilon}
$$

This relation leads to the following equation for running $m^{2}(t)$

$$
\frac{d m^{2}(t)}{d t}=\frac{2 \alpha^{2}}{Q^{2}} m^{2}(t)+\Delta_{m^{2}} m^{2}(t)
$$

where $\Delta_{m^{2}}$ is a scaling dimension of $m^{2}$. To find $\Delta_{m^{2}}$ we consider the term $\frac{m^{2}}{2 \alpha^{2}} e^{\alpha(\sigma+\bar{\sigma})}$ in the action (2) after above scale transformation of the fields $\sigma, \bar{\sigma}$ and action $S$. Scaling dimension of this term is -2 (we take into account that the action is dimensionless), scaling dimension of $e^{\alpha(\sigma+\bar{\sigma})}$ is $2 \alpha$. Hence $\Delta_{m^{2}}=2-2 \alpha$. Therefore, the eq. (8) looks like this

$$
\frac{d m^{2}(t)}{d t}=\left(2-2 \alpha+\frac{2 \alpha^{2}}{Q^{2}}\right) m^{2}(t)
$$


Hence

$$
m^{2}(t)=m^{2} \exp \left(\left(2-2 \alpha+\frac{2 \alpha^{2}}{Q^{2}}\right) t\right)
$$

where $m^{2}=\left.m^{2}(t)\right|_{t=0}$. It is evident that at $2-2 \alpha+\frac{2 \alpha^{2}}{Q^{2}}>0$ we get $m^{2}(t) \rightarrow 0$ i.e the running parameter $m^{2}(t)$ vanishes in infrared limit.

Taking into account everything above we can say that the model with the action

$$
S=\int d^{8} z\left(-\frac{1}{2} \frac{Q^{2}}{16 \pi^{2}} \bar{\sigma} \square \sigma\right)+\left(\frac{\Lambda}{\alpha^{2}} \int d^{6} z e^{3 \alpha \sigma}+\text { h.c. }\right)
$$

is a natural infrared limit of the model (2). The model (11) corresponds to finite quantum theory, i.e. all quantum corrections to effective action in this model will be finite.

6 . Let us consider the effective action corresponding to the model (2) in infrared limit where $\xi_{1}=\xi_{2}=m^{2}=0$. As it has been proved in previous sections the model (2) will be finite in infrared domain and we face a problem of effective action in finite quantum field theory.

Aspects of effective action in supersymmetric models have been investigated in a number of papers (see f.e. [13-19]). 円 We will follow our approach [18, 19] based on superfield generalization of proper-time method.

We suggest that the effective action has the following general structure

$$
\Gamma=\int d^{8} z L+\left(\int d^{6} z \mathcal{L}_{c}+\text { h.c. }\right)
$$

Here $L$ can be called the general effective lagrangian, $\mathcal{L}_{c}$ can be called the chiral effective lagrangian. The $L$ should depend on the field $\sigma, \bar{\sigma}$ and their supercovariant derivatives of any order. In particular there can be a term independent of the derivatives. We will call this term the kahlerian effective potential $K$ (see the discussion in [18]). The $\mathcal{L}_{c}$ is a chiral superfield and therefore it can depend only on $\sigma$ and its space-time derivatives of any order.

To calculate the effective action we will use a technique of loop expansion. In particular, in order to find one-loop contribution to the effective action we should divide the fields into background and quantum and consider a quadratic in quantum fields part of the action depending on background fields (see the details f.e. in [20]).

The effective lagrangians $L(\sigma, \bar{\sigma})$ and $\mathcal{L}_{c}(\sigma)$ can be represented in the form of power expansion in derivatives of $\sigma, \bar{\sigma}$. The lower correction to $L$ should has the form $L \sim$ $\Lambda^{k_{1}} \bar{\Lambda}^{k_{2}} e^{n(\sigma+\bar{\sigma})}$ where $k_{1}, k_{2}$ and $n$ are some numbers. It is turned out that all these numbers can be found exactly without any calculations.

We notice that the action (2) at $\xi_{1}=\xi_{2}=m^{2}=0$ is invariant under transformations $\sigma \rightarrow \sigma+\gamma, \bar{\sigma} \rightarrow \bar{\sigma}+\beta, \Lambda \rightarrow e^{-3 \gamma} \Lambda, \bar{\Lambda} \rightarrow e^{-3 \beta} \Lambda$ where $\gamma$ and $\beta$ are constant independent parameters. The effective action should be also invariant under these transformations (the theory under consideration is finite quantum theory therefore we have no any sources of anomalies there). It means $-3 k_{1}+n=0, k_{2}=k_{1}$. Since the dimension of $L$ is 2 we get $3\left(k_{1}+k_{2}\right)=2$. As a result we find $k_{1}=k_{2}=1 / 3, n=1$. Hence the lower contribution to kahlerian effective potential looks like

$$
K=\rho(\lambda \bar{\lambda})^{1 / 3} e^{\sigma+\bar{\sigma}}
$$

\footnotetext{
${ }^{1}$ We would like to notice that some classical aspects of the first paper in ref.[13] have also been considered in earlier paper [30].
} 
where $\rho$ is some dimensionless constant which can be found only at straightforward calculations.

The lower contribution to $\mathcal{L}_{c}$ should has the form of a linear combination of the terms $\Lambda^{q_{1}} \bar{\Lambda}^{q_{2}} e^{p \sigma} \partial_{m} \sigma \partial^{m} \sigma$ and $\Lambda^{q_{1}} \bar{\Lambda}^{q_{2}} e^{p \sigma} \square \sigma$ where $q_{1}, q_{2}, p$ are some numbers. The dimension of $\mathcal{L}_{c}$ is 3 , hence $3\left(q_{1}+q_{2}\right)+2=3$. The invariance of $\mathcal{L}_{c}$ under above transformations leads to $-3 q_{1}+p=0$ (we use transformations at $\beta=0$ there). As a result $q_{1}=p / 3$, $q_{2}=1 / 3-p / 3$. Hence the lower contribution to $\mathcal{L}_{c}$ looks like this

$$
\mathcal{L}_{c}=\bar{\Lambda}^{1 / 3}\left[\sum_{p_{1}}\left(\frac{\Lambda}{\bar{\Lambda}}\right)^{p_{1}} \rho_{p_{1}} e^{p_{1} \sigma} \partial_{m} \sigma \partial^{m} \sigma+\sum_{p_{2}}\left(\frac{\Lambda}{\bar{\Lambda}}\right)^{p_{2}} \rho_{p_{2}} e^{p_{2} \sigma} \square \sigma\right]
$$

where $\rho_{p_{1}}$ and $\rho_{p_{2}}$ are some dimensionless constants. These constants and the values of $p_{1}$ and $p_{2}$ can be found at straightforward calculations.

The one-loop contrbution $\bar{\Gamma}^{(1)}$ to effective action is given by the relation

$$
\begin{gathered}
\exp \left(i \Gamma^{(1)}\right)=\int D \chi D \bar{\chi} \exp \left(i S^{(2)}[\sigma, \bar{\sigma} ; \chi, \bar{\chi}]\right) \\
S^{(2)}=\int d^{8} z\left(-\frac{Q^{2}}{2(4 \pi)^{2}} \bar{\chi} \square\right)+\left(\frac{9 \Lambda}{2} \int d^{6} z e^{3 \sigma} \chi^{2}+\text { h.c. }\right)
\end{gathered}
$$

Using the trick suggested in ref. [18] one can rewrite the $\bar{\Gamma}^{(1)}$ in the form

$$
\begin{array}{r}
\bar{\Gamma}^{(1)}=-\frac{i}{2} \int_{0}^{\infty} \frac{d \tau}{\tau} s \operatorname{Tr} e^{i \tau \Delta} \\
\Delta=-\frac{Q^{2}}{(4 \pi)^{2}} \square^{2}-9 \Lambda e^{3 \sigma} \frac{\bar{D}^{2}}{4}-9 \bar{\Lambda} e^{3 \bar{\sigma}} \frac{D^{2}}{4}
\end{array}
$$

where $s \operatorname{Tr}$ is a functional supertrace of the operator acting on superfields. We should like to notice especially that the integral over proper time in (15) does not need in regularization since the theory under consideration is finite quantum field theory.

To calculate the one-loop kahlerian effective potential it is sufficient to consider fields $\sigma$ and $\bar{\sigma}$ as independent of space-time coordinates. It leads to

$$
\exp (i \tau \Delta)=\exp \left[i \tau\left(-\frac{9}{4} \Lambda e^{3 \sigma} \bar{D}^{2}-\frac{9}{4} \bar{\Lambda} e^{3 \bar{\sigma}} D^{2}\right)\right] \exp \left(-i \tau \frac{Q^{2}}{(4 \pi)^{2}} \square^{2}\right)
$$

In this approximation the functional supertrace in (15) can be exactly calculated. The final result for kahlerian effective potential has the form

$$
K^{(1)}=c\left(\frac{\Lambda \bar{\Lambda}}{Q^{4}}\right)^{1 / 3} e^{\sigma+\bar{\sigma}}
$$

where $c$ is a positive constant ${ }^{2}$. We see the $K^{(1)}(16)$ completely corresponds to (13) with explicit value for $\rho$.

The calculation of chiral contribution to effective action is more complicated. To do that we can put $\bar{\sigma}=0$ in operator $\Delta$ (15) but we should take into account whole superspace dependence of the chiral superfield $\sigma$. Nevertheless the lower contribution to one-loop chiral effective lagrangian can be calculated in explicit form

$$
\begin{aligned}
\mathcal{L}_{c}^{(1)} & =\frac{\Lambda^{1 / 3}}{Q^{2 / 3}}\left\{\left(c_{1} e^{-\sigma}+c_{2} e^{2 \sigma}+c_{3} e^{-\sigma}+c_{4} e^{-4 \sigma}\right) \times\right. \\
& \left.\times \partial^{m} \sigma \partial_{m} \sigma+\square \sigma\left(c_{3} e^{-\sigma}+c_{4} e^{-4 \sigma}\right)\right\}
\end{aligned}
$$


where $c_{1}, c_{2}, c_{3}, c_{4}$ are some definite numbers 9 . We put for simplicity $\bar{\Lambda}=\Lambda$ there and further. It is evident that the $\mathcal{L}_{c}^{(1)}(17)$ completely corresponds to (14) with explicit values for the $\rho_{p_{1}}, \rho_{p_{2}}$.

We want call attention that both $K(13)$ and $\mathcal{L}_{c}$ (14) display non- polynomial dependence on the couplings $\Lambda$ and $\bar{\Lambda}$.

7. As a result we can write the one-loop effective action $\Gamma^{(1)}$ with lower contributions to $L$ and $\mathcal{L}_{c}$. The $\Gamma^{(1)}$ is a sum of classical action $(2)$ at $\xi_{1}=\xi_{2}=m^{2}=0$ and the quantum corrections $\int d^{8} z K^{(1)}(16)$ and $\left(\int d^{6} z \mathcal{L}_{c}^{(1)}+\right.$ h.c. $)(17)$. Let us investigate a structure of this $\Gamma^{(1)}$ for the superfields $\sigma, \bar{\sigma}$ slowly varying in space-time. It means we can put $\partial_{m} \sigma \approx 0$, $\partial_{m} \bar{\sigma} \approx 0$. If we transform $\sqrt{c}\left(\frac{\Lambda}{Q^{2}}\right)^{1 / 3} e^{\sigma}=\phi, \sqrt{c}\left(\frac{\Lambda}{Q^{2}}\right)^{1 / 3} e^{\bar{\sigma}}=\bar{\phi}$, we obtain

$$
\Gamma=\int d^{8} z \phi \bar{\phi}+\left(\lambda \int d^{6} z \phi^{3}+\text { h.c. }\right)
$$

where $\lambda=Q^{2} c^{-3 / 2}$. This action describes a dynamics of a massless chiral superfield $\phi$ of dimension equal to 1 and coincides with the standard action of Wess- Zumino model. So, we conclde that the model of supergravity chiral compensator leads for slowly varying superfields to standard Wess-Zumino model. One can see that the parameter $\Lambda$ is absent in (18), its role is to transform the dimensionless field $e^{\sigma}$ into the field $\phi$ with dimension 1 .

8. Let us summarize our results. We have formulated a new model of chiral superfield in $N=1, D=4$ flat superspace. This model is generated by superconformal anomaly of matter superfields and can be considered as a simplified model of quantum supergravity in low-energy domain. The features of the model are its complete superfield formulation, non-trivial interactions of chiral and antichiral superfields, presence of five couplings, three of which are dimensionless, and higher (second) derivatives in a kinetic term. The model is a natural supersymmetric generalization of the low-energy quantum gravity model given by Antoniadis and Mottola [6].

The analysis of superficial degree of divergence shows that this model leads to decrease of number of possible divergent structures in comparison with non- supersymmetric model [6]. We have calculated the one-loop superfield counterterms, investigated the equations for running couplings and showed that the model is infrared free and moreover it is finite in infrared limit.

An interesting feature of the model is a non-renormalization theorem according to which the vertex $\Lambda \int d^{6} z e^{3 \sigma}$ has no divergent corrections. Since the superfield $\sigma$ is not renormalized in this model we get that the parameter $\Lambda$ (cosmological constant) is always finite. The analogous vertex including cosmological constant in non-supersymmetric model [6] gets divergent corrections. As a result, unlike non-supersymmetric case, the beta-function for $\Lambda$ is equal to zero in our model. It means the mechanism leading to vanishing of effective cosmological constant suggested in ref. [6] will work only if a supersymmetry is violated.

We have investigated a structure of effective action in the model under consideration in the infrared limit where the model becomes to be finite. We have shown that the effective action is defined by general effective lagrangian and chiral effective lagrangian

\footnotetext{
${ }^{2}$ The explicit forms for the numbers $c, c_{1}, c_{2}, c_{3}, c_{4}(16,17)$ are given in terms of some power series and look like too cumbersome to be given there. The details of calculations leading to $(16,17)$ will be presented somewhere.
} 
and found a generic form of lower contributions to these objects within expansions in derivatives. Generalized proper-time superfield technique allowing to calculate the oneloop corrections to effective action has been developed and lower contributions to effective lagrangians have been calculated in explicit form. We have picked out that the effective action found there is reduced to standard Wess-Zumino model for slowly varying in spacetime superfields.

The various aspects of quantum gravity induced by conformal anomaly of the matter fields have recently been investigated in two- and four-dimensional space-time (see f.e. $[6,21-29])$. We hope that the suggested model allows to consider the analogous and new aspects in quantum supergravity.

\section{Acknowledgements}

The authors are grateful to S.M.Kuzenko and S.D.Odintsov for interesting discussions on some aspects of the work. We are also very thankful to S.J.Gates paying our attention to paper [30] I.L.B. thanks D.Luest, D.Ebert and H.Dorn for their hospitality during his visit to Institute of Physics, Humboldt Berlin University where most part of the work has been fulfilled. This visit was supported by Deutsche Forschungsgemeinschaft under contract DFG-436 RUS 113. The work was supported in part by ISF and Russian government under the grant RI1300 and by Russian Foundation for Basic Research under the project No.94-02-03234.

\section{References}

[1] V.I.Ogievetsky, E.S.Sokatchev. Phys.Lett. B79, 222 (1978).

[2] W.Siegel, S.J.Gates. Nucl.Phys. B147, 77 (1979).

[3] V.I.Ogievetsky, E.S.Sokatchev. Yad.Fiz.(Sov.J.Nucl.Phys) 31, 264(1980); 32, 862 (1980); 32, 870(1980); 32, 1142 (1980).

[4] I.L.Buchbinder, S.M.Kuzenko. Ideas and Methods of Supersymmetry and Supergravity, IOP Publishing Ltd, Bristol and Philadelphia, 1995.

[5] M.T.Grisaru, W.Siegel. Nucl.Phys. B187, 149 (1981); B201, 292 (1982).

[6] I.Antoniadis, E.Mottola. Phys.Rev. D45, 2013 (1992).

[7] S.Ferrara, B.Zumino. Nucl.Phys. B87, 207 (1975).

[8] V.I.Ogievetsky, E.S.Sokatchev. Yad.Fiz. (Sov.J.Nucl.Phys) 28, 852 (1978).

[9] I.L.Buchbinder and S.M.Kuzenko. Nucl.Phys. B274, 563 (1986).

[10] I.L.Buchbinder and S.M.Kuzenko. Nucl.Phys. B308, 162 (1988).

[11] I.L.Buchbinder and S.M.Kuzenko. Phys.Lett. B202, 233 (1988).

[12] S.J.Gates, M.T.Grisaru, M.Rocek, W.Siegel. Superspace or One Thousand and One Lessons in Supersymmetry. Benjamin Cummings, Reading, M.A. 1983.

[13] N.Seiberg. Phys. Lett. B206, 75 (1988); B318, 469 (1993). 
[14] D.Amati, K.Konishi, J.Meurice, G.C.Rossi, G.Veneziano. Phys. Repts. 162, 169 (1988).

[15] P.West. Phys. Lett. B258, 375 (1991).

[16] I.Jack, D.R.T.Jones, P.West. Phys. Lett. B258, 382 (1991).

[17] N.Seiberg, E.Witten. Nucl. Phys. B426, 19 (1994).

[18] I.L.Buchbinder, S.M.Kuzenko, J.V.Yarevskaya. Nucl. Phys. B411, 665 (1992).

[19] I.L.Buchbinder, S.M.Kuzenko, A.Yu.Petrov. Phys. Lett. B321, 372 (1994).

[20] I.L.Buchbinder, S.D.Odintsov, I.L.Shapiro. Effective Action in Quantum Gravity, IOP Publishing Ltd, Bristol and Philadelphia, 1992.

[21] V.G.Knizhnik, A.M.Polyakov, A.B.Zamolodchikov. Mod.Phys.Lett. A3, 819 (1988).

[22] S.D.Odintsov, I.L.Shapiro. Int.J.Mod.Phys. D1, 571 (1993).

[23] E.Elizalde, S.D.Odintsov. Nucl.Phys. B399, 581 (1993).

[24] S.D.Odintsov. Z.Phys. C54, 531 (1992).

[25] S.D.Odintsov, R.Percacci. Mod.Phys. Lett. A9, 2041 (1994).

[26] E.Elizalde, S.D.Odintsov. Phys.Lett. B334, 33 (1994).

[27] I.Antoniadis and S.D.Odintsov. Phys.Lett. B343, 76 (1995).

[28] I.L.Shapiro, G.Cognola. Phys.Rev. D51, 2775 (1995).

[29] I.L.Shapiro, H.Takata. Phys. Rev. D52, 2162 (1995).

[30] S.J.Gates. Nucl. Phys. B238 (1984), 349. 